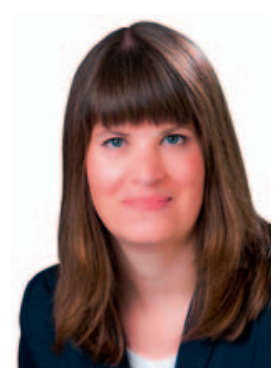

Stephanie Joachim

Koordination «OphthalmoCampus»

\section{Der richtige Blick für Diagnose, Arbeitsalltag und Zukunft}

Liebe Kolleginnen und Kollegen,

in der aktuellen «OphthalmoCampus» im Karger Kompass Ophthalmologie werden wieder interessante Themen aufgegriffen. Dr. Argyrios Chronopoulos und Prof. Dr. Hattenbach berichten von einem interessanten Fall aus ihrer Klinik. Welche Diagnose würden Sie vermuten?

PD Dr. Vinodh Kakkassery aus Rostock gewährt uns einen Einblick in seinen Forschungsaufenthalt in Boston in den USA, während Dr. Sven Schnichels und Marina Löscher aus Tübingen über Organkulturen als Alternativen zu gängigen Tiermodellen oder Zellkulturen berichten. Auch in der Augenheilkunde gewinnen diese Modellsysteme immer weiter an Bedeutung.

Einen ganz anderen Aspekt der ärztlichen Kommunikation, den Humor, bringt uns Dr. Christoph Krause, Anästhesist am Universitätsklinikum Leipzig, näher. Er zeigt uns auf, dass Humor auch der Erhaltung der eigenen Gesundheit dient und bei Konflikten eine wirksame Ressource ist.

Bei der Lektüre des aktuellen «OphthalmoCampus» wünsche ich viel Vergnügen

Mit besten Grüßen,<smiles>CCCCOC(C)CCCC</smiles> 


\section{Blickdiagnose}

\section{Welche Diagnose vermuten Sie?}

Ein 52-jähriger Patient stellt sich mit seit 3-4 Wochen langsam progredienter Visusverschlechterung beim Augenarzt vor. Der Patient hat weder Vorerkrankungen noch eine relevante okuläre Anamnese. Ein bekannter Aderhautnävus am rechten Auge war bisher stabil. Der bestkorrigierte Visus am rechten Auge war 0,3 p und am linken 1,0. An der Spaltlampe stellt sich der Vorderabschnitt bis auf eine leichte seitensymmetrische Linsentrübung unauffällig dar, funduskopisch jedoch zeigte sich am rechten Auge eine ovalförmige juxtafoveale Atrophie mit temporaler RPE-Proliferation und einem Aderhautnävus infranasal der Papille sowie eine Glaskörpertrübung im Sinne einer loka- lisierten hinteren Glaskörperabhebung. Die optische Kohärenztomographie zeigte interessanterweise multiple foveale Zysten sowie eine zentrale neurosensorische Abhebung, vermutlich verantwortlich für die schlechte Funktion. Die Fluoreszenzangiographie zeigte in der Spätphase ein zystoides Makulaödem.

\section{Auflösung online unter: www.karger.com/blickdiagnose-kop-3-2018}

Kontaktadresse: Dr. Argyrios Chronopoulos, Augenklinik, Klinikum der Stadt Ludwigshafen am Rhein, Bremserstraße 79, 67063 Ludwigshafen am Rhein, Deutschland, aris_c@web.de

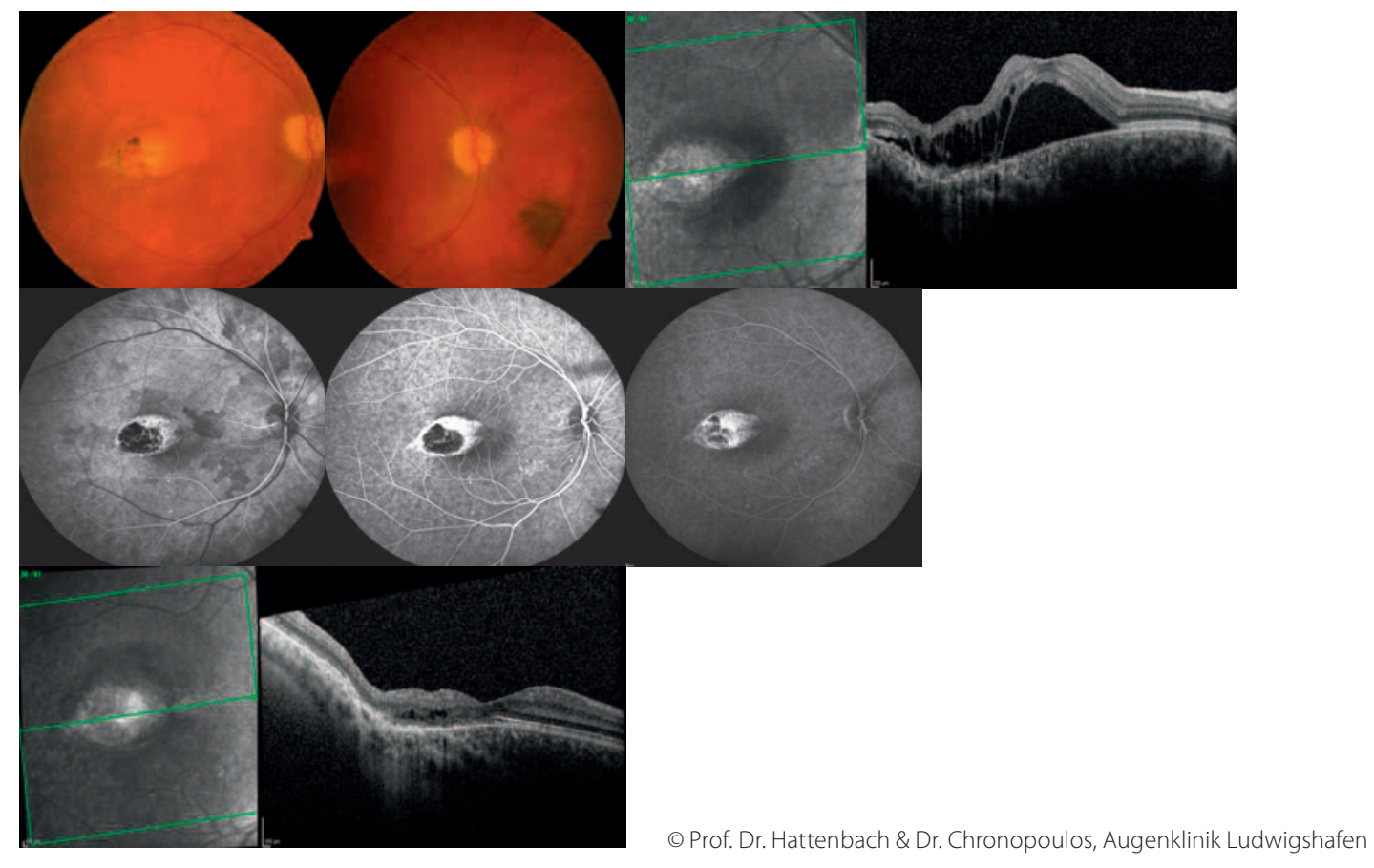




\section{Medizin international}

\section{Forschungsaufenthalt in den USA}

Den Blickwinkel verändern, ein wenig Frischluft schnuppern und neue Ideen für den weiteren Karriereweg gewinnen - das, und noch viel mehr, kann ein Forschungsaufenthalt im Ausland alles bewirken. PD Dr. Vinodh Kakkassery aus Rostock gibt uns einen kurzen, spannenden Einblick und lässt uns an seinen Erfahrungen damit teilhaben.

Herr Dr. Kakkassery, was hat Sie dazu bewogen, ihre Forschung im Ausland fortzusetzen?

Meine Promotionsarbeit habe ich in einem experimentellen Labor in einer Augenklinik in Deutschland durchgeführt. Der Beginn war einfach ein Sprung ins kalte Wasser mit wenig Vorwissen. Ich habe dort einen Assistenzarzt kennengelernt, der zwei Jahre in England im Forschungslabor verbracht hat. Das war ein anderes Niveau, der konnte mit den gestandenen Leuten im Labor diskutieren. Mir war klar, dass man während eines Forschungsaufenthalts schneller und qualitativ hochwertiger lernt. Das hat einen unheimlichen Reiz auf mich ausgeübt. Ich wollte ins Ausland.

\section{Beschreiben Sie, wie Sie den Aufenthalt im Ausland vorbereitet haben.}

Der Bewerbungsverlauf war sicher ein we-

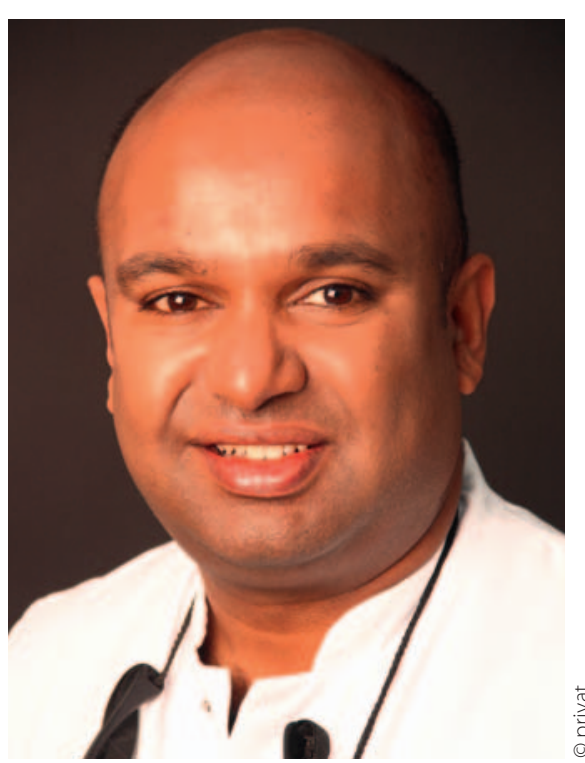

«Ein Auslandsaufenthalt ist sicher für neugierige Menschen und die, die noch Antworten im Leben suchen, eine absolute Bereicherung.» habe ich verschiedene neue molekularbiologische Methoden gelernt und war in der Lage beim Versuchsaufbau die Strukturen weiter zu verbessern. Ebenso konnte ich nach dem Aufenthalt Forschungsprojekte planen, durchführen und die Ergebnisse analysieren und bewerten. Und ich habe publiziert - das hätte allerdings mehr sein dürfen. Als Mensch war die Zeit eine absolute Bereicherung. Ich habe einen neuen Blickwinkel erhalten und war danach besser in der Lage, Systeme zu bewerten und Abläufe und Ereignisse in der Karriere einzuordnen und zu verstehen.

Wie unterscheidet sich die Arbeit in den USA von den Gegebenheiten in Deutschland?

Da ich in Deutschland hauptsächlich in der Klinik tätig war und nur nebenbei geforscht habe, in Boston dagegen die Forschung im Mittelpunkt stand, ist beides nicht wirklich miteinander zu vergleichen. Die Zeit in Boston hat mir sehr viel Spaß bereitet und die Fokussierung auf die Forschung war der wesentliche Unterschied, die einfach wertvoll für meine Entwicklung war.

\section{Was hat Sie am meisten während Ihres USA-Aufenthalts herausgefordert?}

Sicherlich im ersten halben Jahr eine gewisse Einsamkeit, da man sich in einem fremden Land mit einer relativ ungewohnten Sprache erstmal zurechtfinden und Leute kennenlernen muss. Außerdem das tägliche ungewohnte Leben an sich sowie die kulturellen Unterschiede, die sich im Umgang mit Menschen beruflich und auch privat gezeigt haben.

Was haben Sie aus dem Auslandsaufenthalt für Ihre jetzige Arbeit an der Augenklinik in Rostock mitnehmen können?

Das eigenständige Denken und der Wunsch zu gestalten sind mir in Boston «eingepflanzt» worden. Ebenso bin ich als Wissenschaftler «geschliffen» worden und habe mich da zu einer autarken Person entwickelt. Auch neue Arbeitsumfelder zu erfassen und zu verstehen ist eine während des Auslandsaufenthalts erlernte Eigenschaft, die immer hilft.

\section{Würden Sie Ihren Kollegen einen Auslandsaufenthalt empfehlen? Wenn ja, warum?}

Die Chance, sich menschlich weiterzuentwickeln, ist einfach sensationell. Sehr wichtig ist, dass man sich parallel dazu beruflich entfaltet. Ein Auslandsaufenthalt ist sicher für neugierige Menschen und die, die noch Antworten im Leben suchen, eine absolute Bereicherung. 
PD Dr. Vinodh Kakkassery arbeitet heute als Oberarzt an der Klinik und PoliklinikfürAugenheilkundein Rostock. SeineWeiterbildung zum Facharzt für Augenheilkunde leistete er am Zentrum für Augenheilkunde des Universitätsklinikums Essen ab. Neben dem Postdoctoral Fellowship am Schepens Eye Research Institute an der Harvard Medical School, Boston, USA sammelte PD Dr. Kakkassery als Schiffsarzt auf dem Toppsegelschoner «Thor Heyerdahl» weitere Auslandserfahrungen. Während all dieser Schritte auf seinem Lebensweg konnte er sich immer auf die Unterstützung und weisen Rat seiner Eltern verlassen.
Welche Empfehlungen würden Sie jemandem mit auf den Weg geben, der sich für einen Auslandsaufenthalt interessiert?

Sich zu trauen und sich auf seinen Instinkt zu verlassen. Und sich in schwierigen Situationen an dem roten Faden im Leben orientieren, den man von seinen Eltern mitbekommen hat.

\section{Wir danken Ihnen herzlich für das Interview!}

Kontaktadresse: PD Dr. Vinodh Kakkassery, Klinik und Poliklinik für Augenheilkunde, Universitätsmedizin Rostock, Doberaner Straße 140, 18057 Rostock, vinodh.kakkassery@gmail.com.

\section{Ophthalmologie kompakt \\ Alternativen zu Tiermodellen in der augenheilkundlichen Forschung}

Die ophthalmologische Forschung, benötigt, wie jedes andere Forschungsgebiet auch, Modelle, um neue Therapien zu testen und Degenerationsprozesse zu verstehen. Die Erforschung erfolgt dabei hauptsächlich in Tierversuchen. Dies ermöglicht physiologische sowie pathophysiologische Prozesse im lebenden System zu untersuchen, was entscheidend ist, um Wirksamkeit und Verträglichkeit von neuen Therapien zu untersuchen. Gleichzeitig sind Tierversuche regulatorische Voraussetzung, bevor klinische Studien am Menschen durchgeführt werden können. Tierversuche sind allerdings durchaus kritisch zu hinterfragen. Nicht nur der zeitliche Aufwand und die hohen Kosten spielen hier eine Rolle, vor allem auch ethische Bedenken sind von hoher Relevanz. Weiterhin zeigt sich nun gehäuft, dass die Übertragbarkeit von den Standardtiermodellen (hauptsächlich Mausmodelle) auf den Menschen oft nicht möglich ist. Ursachen hierfür sind u.a. die fehlende Makula, ein anderes Verhältnis von Photorezeptoren und auch ein viel geringerer Glaskörperraum.
Diese Erwägungen haben in den letzten Jahren und Jahrzehnten zur Entwicklung von Organkulturmodellen geführt, die die Vorteile von reinen Zellkulturexperimenten und In-vivo-Experimenten verbinden und damit im Sinne des 3R-Prinzips (Replace, Reduce, Refine) den Einsatz von Tierversuchen verringern und zumindest teilweise auch ersetzen können. Dabei gibt es verschiedene Ansätze diese Modelle herzustellen: Neben der Organkultur durch postmortale Entnahme des Gewebes (Abb. 1), gibt es auch Ansätze, den Gewebeverband künstlich herzustellen in Form von Organoiden, 3D-Zellkulturen oder Organ-on-a-Chip-Modellen. Der direkteste und einfachste Weg bei der Organkultur ist die postmortale Entnahme des Gewebes. Hierbei ist prinzipiell zwischen Kulturen aus Versuchstieren und denen aus Schlachthoftieren zu unterscheiden. Während für erstere weiterhin Tiere extra gezüchtet und getötet werden müssen, fallen letztere quasi als Abfallprodukt der Lebensmittelindustrie an. Hierbei handelt es sich vornehmlich um

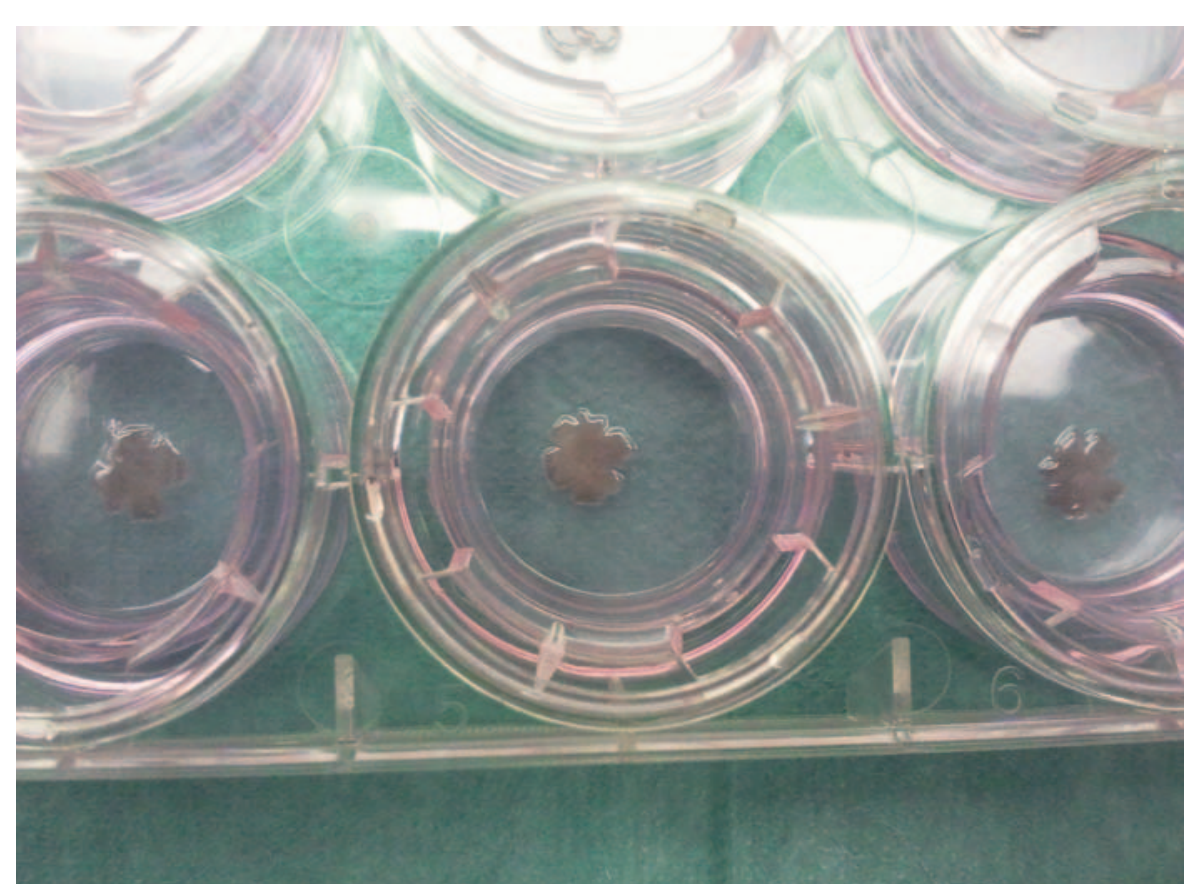

Abb. 1. Retinale Organkultur gewonnen aus Rattenaugen. 
Abb. 2. Pubmed-Recherche nach den Begriffen «corneal culture model» und «retinal culture model». Dargestellt ist die kumulierte Anzahl der Publikationen im jeweiligen Jahr.

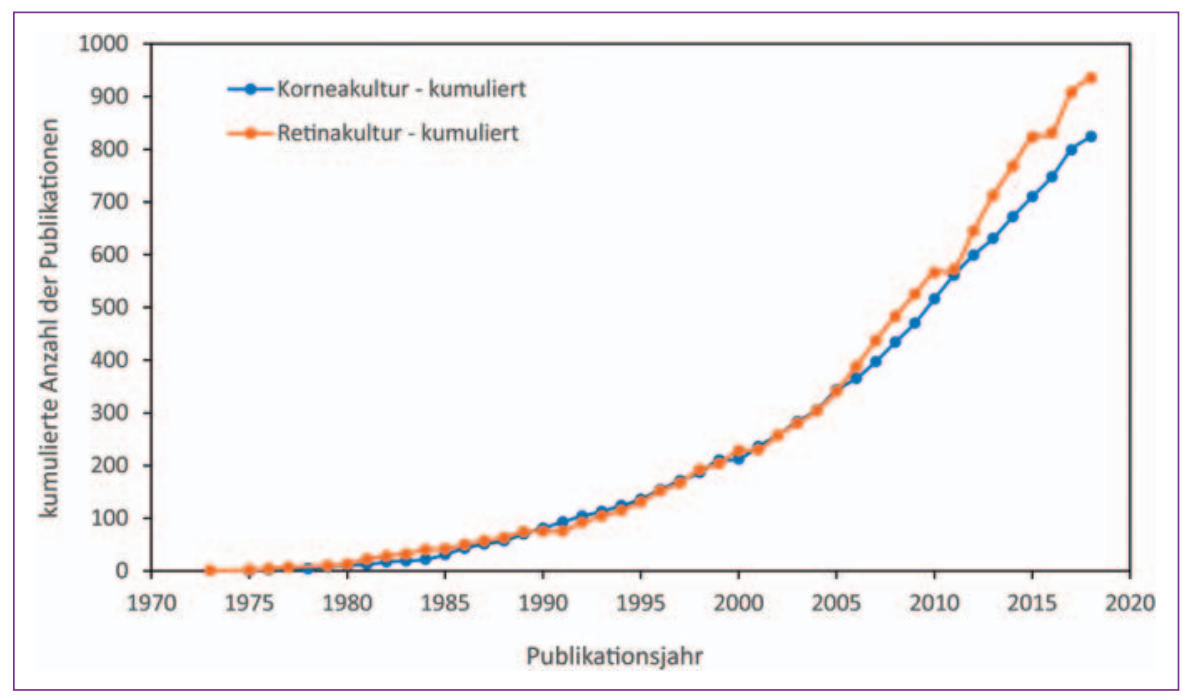

Rinder- und Schweineaugen, die dem menschlichen Auge in Größe und Morphologie wesentlich ähnlicher sind als die Nageraugen der verwendeten In-vivo-Modelle. Neben der Kosten- und Bürokratieeinsparung durch die Verwendung von Augen vom Schlachthof, ist auch die zeitliche Einsparung enorm, da die Augen quasi täglich anfallen und nicht extra darauf gewartet werden muss, bis die Versuchstiere das entsprechende Alter für die Untersuchung erreicht haben. Zudem können aufgrund der Größe der Augen mehrere Proben aus einem Auge gewonnen werden, was die Vergleichbarkeit erhöht.

In der Augenheilkunde sind schon einige Korneamodelle etabliert und zum Teil von den regulatorischen Behörden anerkannt, darunter der EpiOcular'M Eye Irritation Test (EIT), der SkinEthic ${ }^{T M}$ Human Corneal Epithelium (HCE) Eye Irritation Test (EIT), der Isolated Chicken Eye (ICE) Test, der Bovine Corneal Opacity and Permeability (BCOP) Test, der Flourescein Leakage (FL) Test und der Short Time Exposure (STE) Test [1-6].

Retinale Modelle sind wesentlich schwieriger zu etablieren, da es sich bei der Netzhaut um ein komplexes Gewebe mit verschiedenen neuronalen und nicht-neuronalen Zellen handelt. Trotzdem gibt es in jüngerer Zeit mehrere Ansätze, ein retinales Organkulturmodell zu entwickeln [7-20].

Die Zahl der Publikationen über Organkulturen hat in den letzten Jahren stetig zugenommen (Abb. 2) und das Interesse ist auch seitens der Pharmaindustrie sehr hoch. Vor allem in der Erforschung retinaler Erkrankungen, deren Behandlungsspektrum noch immer viel Optimierungsbedarf bietet, bergen gut etablierte und charak- terisierte Organmodelle enormes Potential, Entwicklungszeit und -aufwand von neuen Therapeutika zu verringern und Pathomechanismen besser zu verstehen.

Die bereits zertifizierten Korneamodelle lassen hoffen, dass in naher Zukunft auch retinale Ex-vivo-Modelle zertifiziert werden und somit nicht nur zur erfolgreichen Therapieentwicklung, sondern auch zur Einsparung von Tierversuchen beitragen.

\section{Literatur}

1 OECD. Test No. 437: 2009;DOI:10.1787/9789264076303-en.

2 OECD. Test No. 438: 2009;DOI:10.1787/9789264076310-en.

3 OECD. Test No. 460: 2017;DOI:10.1787/9789264185401-en.

4 OECD. Test No. 491: 2017;DOI:10.1787/9789264242432-en.

5 OECD. Test No. 492: 2017;DOI:10.1787/9789264242548-en.

6 Lee M, et al.: Toxicol res 2017;33:191-203.

7 Hurst J, et al.: Altern Lab Anim 2017;451:11-25

8 Januschowski K, et al.: J Vis Exp 2015;DOI:10.3791/52270.

9 Kuehn S, et al.: Altern Lab Anim 2016;44:557-568

10 Schnichels S, et al.: Biol Open 2017;6:1056-1064.

11 Schultheiss M, et al.: PLoS One 2016;11:e0148616.

12 Volkner M, et al.: Stem Cell Reports 2016;6:525-538.

13 Shirazi Fard S, et al.: J Vis Exp 2015;DOI:10.3791/53202.

14. Roska B, Sahel JA: Nature 2018;557:359-367.

15. Li Y, et al.: Tissue Cell 2018;51:1-7.

16. Valdes J, et al.: Altex 2016;33:459-464.

17. Osborne A, et al.: Exp Eye Res 2016;143:28-38.

18. Peynshaert K, et al.: Adv Drug Deliv Rev 2018;126:44-57.

19. Ghosh F, et al.: Exp Eye Res 2018;173:13-23.

20. Taylor L, et al.: J Neuroimmunol 2016;298:117-129.

Kontaktadresse: Dr. Sven Schnichels \& Marina Löscher, Universitätsaugenklinik Tübingen, Elfriede-Aulhorn-Straße 7, 72076 Tübingen, Deutschland, sven.schnichels@med.uni-tuebingen.de. 


\section{Arzt-Patienten-Kommunikation}

\section{Ein Appell für mehr Humor im ärztlichen Berufsalltag!}

Humor ist in aller Munde: bei Jan Böhmermann oder der Serie «Die Anstalt», bei Otto Waalkes oder Loriot, bei Dr. Eckart von Hirschhausen oder Vince Ebert. Aber: Gibt es auch kompetente und humorvolle praktizierende Ärztinnen und Ärzte? Denn ein Blick in den ärztlichen Berufsalltag legt nahe, dass der Humor proportional zum Aufstieg auf der Karriereleiter häufig abnimmt.

Die Kombination aus humorvoll und kompetent kennt meist nur ein Kollege, der eine Kollegin hatte, die einen Kollegen kennt, der so ist. Aber selbst erlebt, hat das noch niemand. Nur bei sich selbst natürlich - denn sind wir nicht alle unglaublich feinfühlig und haben bestechenden Witz?

Wenn wir uns jetzt unter den Kollegen umschauen, gibt es ein paar, mit denen können wir lachen. Ein Großteil taugt zur professionellen Zusammenarbeit und der Rest der Gaußschen Verteilung ist gelinde gesagt eine Zumutung. Aber alle glauben bzw. sind überzeugt davon, in Sachen Kommunikation und Humor etwas drauf zu haben. Woran liegt das? Berufs- und Lebenserfahrung? Aus- und Fortbildungen in ärztlicher Kommunikation? Oder weil wir von Kindesbeinen an sprechen gelernt haben?

«Humor ist der Knopf, der verhindert, dass uns der Kragen platzt.»

Joachim Ringelnatz

Wenn wir ehrlich sind, haben die meisten von uns einen Strahlenschutz-Kurs, einen Abdomen-Ultraschall-Kurs oder einen BLSKurs besucht. Aber gilt das auch für einen Kurs in ärztlicher Kommunikation oder noch spezieller zum Thema «Humor in der Medizin »? Fehlanzeige! Dabei führen Ärztinnen und Ärzte gut 200000 Gespräche im Laufe ihrer Karriere. In einem Bereich, der hoch-

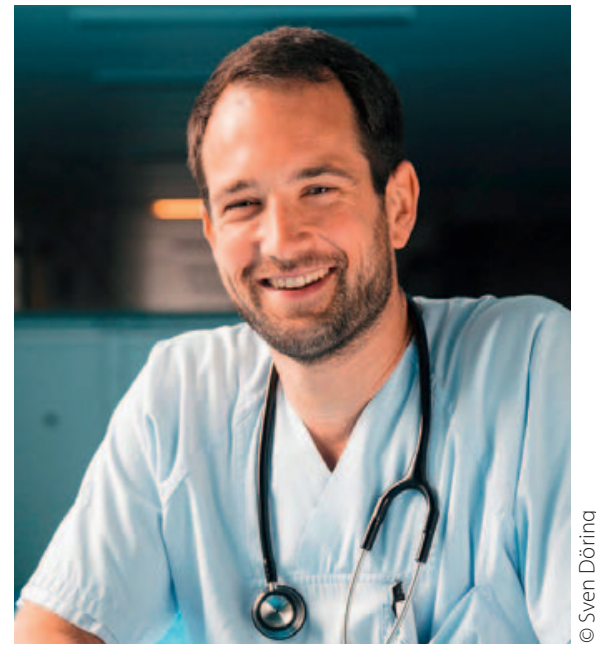

«Denn ein Blick in den ärztlichen Berufsalltag zeigt, dass der Humor proportional zum Aufstieg auf der Karriereleiter abnimmt.» schen Kontext u.a. die kardiopulmonale Physiologie positiv beeinflusst, dass er ablenken kann, die Perspektive ändert und damit Ängste reduziert.

"Kommen eine Seele und ein Körper gemeinsam ins Krankenhaus. Sagt die Seele zum Körper: «Geh ruhig vor. Dich verstehen sie hier besser.ı»

Ulrich Schaffer

Im medizinischen Kontext fehlt es nicht an Humor. Er bedient nur zu schnell Schenkelklopfer, wird zu plump oder grenzt unsere PeerGroups ab. Chirurgen und Anästhesisten können davon ein Lied singen. «Wie nennt man das grüne Tuch zwischen OP-Feld und Beatmung? Blut-Hirn-Schranke!» Das macht zwar kurzfristig Spaß, verbessert langfristig aber die Zusammenarbeit nicht.

Feinsinnig seinen humorvollen Fingerabdruck im stressigen Berufsalltag zu hinterlassen, das ist eine ärztliche Kunst, die begeistert. Mein Gegenüber humorvoll aufzuwerten, ein Ventil für Druck und Stress zu haben und sich selbst vor dem Ausbrennen zu bewahren, das alles kann ein spezielles Training bei «Arzt mit Humor» bewirken. Denn den Humor bringen Sie mit. Sie müssen inn nur bewusst nutzen und die Situation nicht dem Zufall überlassen. Nicht aufgesetzt und künstlich, sondern authentisch und echt. Das sind dann Sie! Wenn die eigene Leistungsfähigkeit steigt, die Freude im Arztberuf wiederkommt und Humor zur Erhaltung der eigenen Gesundheit oder im Konflikt eine wirksame Ressource wird, dann haben Sie wohl ein Training von «Arzt mit Humor» besucht und es hat Sie überzeugt. Das nächste Training «Humor auf Rezept? Die Dosis bringt den Erfolg!» für Ärztinnen und Ärzte findet am 15. und 16.03.2019 in Leipzig statt. Anmeldung und weitere Inforsensibel für Missverständnisse, Kompetenzgerangel und anstrengende Patienten ist. Nicht schlimm, das kann man ändern. Die Initiative «Arzt mit Humor» hat sich auf die Fahnen geschrieben, den Humor auch im Krankenhaus oder der Arztpraxis salonfähig zu machen. Denn die Gaußsche Verteilung der Kolleginnen und Kollegen mit Humor entsteht nicht, weil das Gegenüber keinen hat, sondern weil es Ihren Humor nicht teilt oder eben einen anderen hat. Dasselbe gilt übrigens auch für Patienten, ihre Angehörigen und das Pflegepersonal.

Wir wundern uns manchmal, wie viel «Arzt» ein Patient verträgt? Weg von der rein medizinischen Sichtweise interessiert mich, wie viel Humor haben meine Patienten, meine Kollegen, das Pflegepersonal noch bzw. wie viel geht noch? Oder warum ist der Humor abhandengekommen? Oder kann Humor meinem Problem schaden? Man weiß, dass sich in entspannten Situationen besser lernen lässt, dass Humor die Aufmerksamkeit erhöht und er im medizini- mationen unter www.arztmithumor.de.

Dr. Christoph Krause ist Anästhesist am Universitätsklinikum Leipzig und fährt als Notarzt durch die Stadt. Vor Jahren hat er zusammen mit dem «Deutschen Institut für Humor» die Initiative «Arzt mit Humor» ins Leben gerufen, weil der Berufsalltag nicht immer die wahre Freude war, wenn es um den Umgang zwischen der Ärzteschaft, den Studierenden, dem Pflegepersonal, den Patienten und den Angehörigen ging. Wohl wissend, dass es im Gesundheitswesen Faktoren gibt, die den Idealismus verschrecken, hält er an der Vision fest, gute ärztliche Kommunikation und Hochleistungsmedizin miteinander zu verbinden.

Kontaktadresse: Dr. Christoph Krause, Deutsches Institut für Humor $(\mathrm{DIH})^{\circledR}$ Feuerbachstraße 26, 04105 Leipzig, Deutschland, christoph.krause@arztmithumor.de. 